\title{
FAUT-IL INTÉRESSER LES MÉDECINS POUR LES MOTIVER ? UNE ANALYSE CRITIQUE DU PAIEMENT À LA PERFORMANCE MÉDICALE
}

Nicolas da Silva

\author{
La Découverte | «Revue du MAUSS »
}

2013/1 n 41 | pages 93 à 108

ISSN 1247-4819

ISBN 9782707176455

Article disponible en ligne à l'adresse :

http://www.cairn.info/revue-du-mauss-2013-1-page-93.htm

\section{Pour citer cet article :}

Nicolas da Silva, «Faut-il intéresser les médecins pour les motiver ? Une analyse critique du paiement à la performance médicale », Revue du MAUSS 2013/1 (n 41), p. 93-108.

DOI 10.3917/rdm.041.0093

Distribution électronique Cairn.info pour La Découverte.

(C) La Découverte. Tous droits réservés pour tous pays.

La reproduction ou représentation de cet article, notamment par photocopie, n'est autorisée que dans les limites des conditions générales d'utilisation du site ou, le cas échéant, des conditions générales de la licence souscrite par votre établissement. Toute autre reproduction ou représentation, en tout ou partie, sous quelque forme et de quelque manière que ce soit, est interdite sauf accord préalable et écrit de l'éditeur, en dehors des cas prévus par la législation en vigueur en France. Il est précisé que son stockage dans une base de données est également interdit. 
Faut-il intéresser les médecins pour les motiver?

\author{
Une analyse critique du paiement \\ à la performance médicale ${ }^{1}$
}

\author{
Nicolas da Silva
}

\title{
Introduction
}

En France comme ailleurs, les réformes du secteur de la santé prennent appui sur des dispositifs marchands ayant pour but de modifier le comportement des individus. Elles s'inspirent de la théorie économique standard où les individus sont supposés être opportunistes, réticents à l'effort et intéressés. L'idée est alors de promouvoir, tant du côté de l'offre (les médecins et les hôpitaux) que de la demande de soins (les patients), un comportement entrepreneurial qui incite à la responsabilisation financière tout en cherchant à améliorer la qualité des soins.

En médecine de ville, cela s'est traduit depuis 2009 par la possibilité pour un médecin français de signer le contrat d'amélioration des pratiques individuelles (CAPI) et d'être rémunéré à la performance. Ce mécanisme prévoit un intéressement financier lorsque des objectifs prédéfinis sont atteints. Le cas de la

1. Je tiens à remercier chaleureusement Philippe Batifoulier et Alain Caillé qui ont grandement contribué à l'amélioration des versions antérieures de ce texte. Je reste cependant le seul responsable des imperfections subsistantes. 
France s'inscrit dans une dynamique internationale qui est pourtant à questionner. Aussi bien théoriquement qu'empiriquement, la démonstration du bien-fondé de ce mode de rétribution reste à faire. Le développement de ce type de dispositif appelle donc une discussion critique.

Il semblerait en effet que les dispositifs d'intéressement n'améliorent pas ou peu la performance. Les médecins réagissent de façon inattendue aux incitations économiques et les résultats obtenus sont rarement à la hauteur des résultats escomptés par la puissance publique (1). Cet état de fait invite à questionner les motivations médicales. L'appropriation ou le rejet des mécanismes de paiement à la performance, ainsi que leurs faibles résultats, disent quelque chose de leur motivation. Les médecins ne paraissent pas exclusivement intéressés par l'intéressement (financier) et la rationalité du dispositif n'est pas là où on l'attend (2).

\title{
1. La performance du paiement à la performance
}

\author{
Quelle définition de la performance \\ et de sa rémunération en médecine?
}

Si l'on suit la théorie économique standard, le médecin est un individu intéressé comme les autres. La particularité de son activité ne change pas l'analyse de son comportement. Il reste un Homo xconomicus. À ce titre, il cherche dans toutes circonstances à tirer la couverture à lui et à satisfaire son bien-être personnel. On pourrait croire que la théorie économique a relativisé ce point de vue dans le cas du médecin qui, au moins dans l'imaginaire collectif, n'est pas considéré comme l'archétype de l'individu froid et cynique qui n'a que l'égoïsme pour mot d'ordre. Il n'en est rien et c'est même le contraire [Batifoulier, 2004].

L'accent étant mis sur l'opportunisme du médecin, le problème du théoricien est de trouver une méthode de rémunération capable de le contrer. Il faut limiter son pouvoir discrétionnaire. Or toujours selon l'analyse standard, les modes de rémunération traditionnels du médecin (paiement à l'acte, salariat et capitation) lui fournissent des incitations perverses car elles alimentent son opportunisme naturel. 
Le mode de rémunération optimal est alors le paiement à la performance (pay for performance ou P4P). En effet, le médecin étant réticent à l'effort et intéressé, il faut l'inciter à l'effort. Il est nécessaire de relier strictement la performance observée avec la rémunération. C'est un mécanisme d'intéressement. En payant le médecin au niveau de la production observée, on s'assure de ne pas être spolié au cas où il ne produirait pas d'effort. Afin de réorienter le comportement du médecin, il faut contrer ses tendances naturelles à l'opportunisme par l'incitation financière.

Ce discours économique a un impact sur l'ensemble des politiques publiques. Ainsi, selon le rapport Bureau et Mougeot [2007] sur la performance de la gestion publique en France, le régulateur étant incapable de gérer convenablement les deniers publics, il est nécessaire [c'est un « impératif démocratique », p. 7] de réorienter l'action publique vers la performance. Dans le cadre de la santé, à l'hôpital comme en médecine de ville, il devient nécessaire d'intéresser les acteurs à la performance. C'est pourquoi on observe aujourd'hui une dynamique internationale de mise en pratique du P4P. Attardons-nous particulièrement sur les systèmes de $\mathrm{P} 4 \mathrm{P}$ français et britannique. Ce travail d'approfondissement est nécessaire pour répondre à une question centrale : que veut dire « performance » dans l'expression « paiement à la performance »? Ces deux cas vont nous permettre de voir que cette définition n'a rien d'évident ni de consensuel.

Au Royaume-Uni, le National Health Service a introduit dès 2004 un programme de P4P nommé Quality Outcome Framework $\left(\mathrm{QOF}^{2}\right)$. Ce nouveau mode de rémunération a pour objectif, d'une part, d'améliorer la qualité des soins, et d'autre part, de réorganiser la gestion et l'administration des cabinets médicaux. La performance est mesurée (et donc définie) par cinq types d'indicateurs : clinique, organisation du cabinet, services additionnels, satisfaction des patients et performance globale. Par exemple, pour le domaine clinique, plus de quatre-vingt indicateurs portent tant sur des domaines cliniques (hypertension, diabète, asthme etc.) que de santé publique (obésité, tabagisme etc.). Chaque indicateur fixe un pourcentage minimum de patients qui doivent être traités pour

2. Pour une description détaillée du QOF, on pourra se référer au récent rapport de l'Inspection générale des affaires sociales [Bras et Duhamel, 2008]. 
percevoir la rémunération à la performance. Au-dessus de ce seuil minimal, plus le cabinet augmente son pourcentage de patients traités, plus la rémunération est importante (jusqu'à un seuil maximal). À ces différents objectifs, sont attribués des points qui eux-mêmes correspondent à une rémunération supplémentaire pour les cliniques. Tous objectifs confondus, les cabinets peuvent, pour un taux de réalisation de $100 \%$, obtenir près de 1000 points. Financièrement, la valeur du point est de 133,76 livres sterling, soit un maximum de 133760 livres sterling de rémunération à la performance en cas d'atteinte complète des objectifs pour l'année en cours.

En France, le dispositif de P4P, en vigueur dès 2009, s'appelle le contrat d'amélioration des pratiques individuelles $\left(\mathrm{CAPI}^{3}\right)$. Ce contrat individuel a pour objectif, sur la base du volontariat, d'introduire une dose de $\mathrm{P} 4 \mathrm{P}$ dans la rémunération du médecin ${ }^{4}$. Le CAPI prévoit des contreparties financières pour les médecins qui parviennent à atteindre des objectifs prédéfinis dans deux « champs »: « Dépistage et prévention; suivi des pathologies chroniques » (dépistage et suivi) et «Optimisation des prescriptions » (optimisation). À l'intérieur de ces champs, seize indicateurs sont définis pour mesurer la performance des médecins. À condition d'atteindre au moins $25 \%$ de réalisation globale, la rémunération à la performance est égale à 7 euros par patient multiplié par le taux de réalisation global. Par exemple, dans le champ optimisation, la performance du médecin est calculée à partir d'un indicateur de taux de prescription d'antibiotiques dans le répertoire officiel des génériques. Si le taux de prescription en nombre de boîtes d'antibiotiques figurant dans le répertoire des génériques dans la prescription totale des génériques est supérieur ou égal à $90 \%$ (taux de réalisation cible), alors le médecin est dit performant et peut percevoir la rémunération correspondante.

La comparaison des mécanismes britanniques et français met en évidence les difficultés liées à la définition de la performance du

3. Pour une description plus complète du CAPI, voir la décision du 9 mars 2009 parue au Journal officiel de la République française le 21 avril 2009.

4. Notons dès maintenant que la Convention médicale de juillet 2011 a élargi le P4P à tous les médecins. Cet élargissement s'est fait sur la base du CAPI mais avec de nouveaux indicateurs. Nous présentons ici uniquement le CAPI puisque les données de la nouvelle convention ne sont pas encore disponibles. 
médecin. Il y a autant de définitions que d'indicateurs. Cependant, les pouvoirs publics se sont engagés sur la voie de P4P. Il convient alors d'évaluer ce dispositif empiriquement.

\section{Des médecins dorénavant performants?}

Une évaluation des dispositifs de P4P

L'évaluation des politiques de $\mathrm{P} 4 \mathrm{P}$ peut se faire à partir de trois critères : efficacité, efficience et effets pervers. L'efficacité d'une politique économique se définit comme l'atteinte des objectifs fixés. Dans le cas de la santé, nous avons vu que les objectifs sont différents selon les pays. Mais, globalement, le P4P a deux objectifs principaux : réduire les dépenses de santé et améliorer la qualité des soins. La politique de P4P peut alors être qualifiée d'efficace si et seulement si elle atteint ses objectifs. Or le point d'accord majeur entre les différentes revues de la littérature sur le sujet porte sur l'incapacité actuelle à prouver un effet strictement positif (ou négatif) du P4P tant sur la dimension qualitative qu'économique [Van Herck et al., 2010]. La qualité des données et des méthodes statistiques est un rempart difficilement surmontable pour donner des résultats dénués d'ambiguiité. Du point de vue de l'efficacité clinique (dimension qualitative), les résultats des expériences varient de négatifs à très positifs, en passant par aucun effet ou faiblement positifs. Du point de vue de l'économie en ressources, la revue de Van Herck et al. [2010] fait apparaître des résultats soit faiblement positifs soit négatifs.

Dans le cas du QOF britannique, même si, depuis la mise en place de ce nouveau mode de rémunération des cliniques, on observe une amélioration globale de la qualité des soins, il n'est pas possible de l'attribuer au P4P. Les bonus attribués au titre de la performance peuvent être en grande partie considérés comme un effet d'aubaine, l'amélioration de la qualité étant expliquée par d'autres facteurs. Dès lors, il est possible de qualifier l'impact du QOF sur la qualité des soins de «modeste » [Bras et Duhamel, 2008]. Cela ne veut toutefois pas dire que les médecins sont en cause mais simplement que les incitations viennent rémunérer un travail déjà fait ou qui aurait été fait quoi qu'il arrive [Galvin, 2006].

Ces résultats qualitatifs permettent aussi de mettre en exergue les résultats financiers du QOF tant sur le plan de l'efficacité que 
de l'efficience. D'abord, sur le plan de l'efficacité, il faut noter que le projet politique de Tony Blair avec le QOF était de revaloriser le budget global du National Health Service (NHS), celui-ci étant jugé trop faible. L'objectif initial était d'augmenter le budget du NHS de $41 \%$ entre l'exercice 2002-2003 et l'exercice 2005-2006, soit une augmentation de 1 milliard de livres sterling pour le seul QOF. Le résultat final étant une augmentation de $64 \%$ du budget du NHS (près de 23 points de plus que prévu), on peut raisonnablement qualifier la politique de $\mathrm{P} 4 \mathrm{P}$ d'inefficace sur la question de l'allocation des ressources ${ }^{5}$. Plus globalement, l'efficacité du P4P est limitée.

Mais l'atteinte des objectifs n'est pas le seul critère d'évaluation. En plus d'être efficace, la nouvelle politique publique se justifie par son efficience, c'est-à-dire qu'elle doit atteindre ses objectifs à moindre coût. Il faut alors se demander si une autre politique que le $\mathrm{P} 4 \mathrm{P}$ aurait pu obtenir les mêmes résultats à moindre coût. Au regard de résultats qualitatifs et économiques assez minimes, le coût engendré ne peut apparaître que prohibitif. Entre l'exercice 2002-2003 et l'exercice 2005-2006, le surcoût du QOF s'élevait à plus de 500 millions de livres sterling. On peut raisonnablement supposer qu'à la vue de résultats aussi modestes, tant sur le plan économique que qualitatif, d'autres types de régulation auraient pu être mis au point pour un coût inférieur. L'efficience du P4P est, pour le moins, douteuse.

Enfin, la politique publique de $\mathrm{P} 4 \mathrm{P}$ doit être jugée à l'aune de ses effets pervers. L'un des premiers effets pervers que l'on peut mentionner est l'existence d'un phénomène de sélection des patients où les médecins peuvent exclure de leurs indicateurs certains patients jugés peu coopératifs ou trop malades parce qu'ils risquent de dégrader leur performance. Dans le cas du QOF, les médecins ont la possibilité, dans quelques cas, d'exclure certains patients du calcul qui détermine le niveau de leur rétribution au titre du P4P. Doran et al. [2006] démontrent qu'une augmentation du taux d'exclusion (nombre de patients exclus des critères de performance dans la population totale) de $1 \%$ entraîne une augmentation de la performance de $0,31 \%$. L'exclusion d'une partie de la patientèle

5. Rappelons qu'ici inefficace signifie simplement l'atteinte des objectifs fixés. Il ne s'agit pas de condamner l'augmentation du budget, bien au contraire, mais de constater que le résultat final est différent de l'objectif initial. 
pose alors un problème de justice sociale. Une étude ultérieure montre que, entre 2005 et 2006, $50 \%$ des médecins ont exclu 5,3\% des patients ou plus, ceci représentant un gain supplémentaire pour les cliniques (ou un coût supplémentaire pour le NHS) de 1,5\% [Doran et al., 2008].

L'introduction du P4P pose également un problème de définition de la qualité des soins [Peckham et Wallace, 2010]. Peut-on réduire la définition de la qualité aux seuls critères de performance ? Ces derniers sont d'ailleurs assez différents selon les systèmes de santé qui les imposent. Dans le cas français, la performance se définit par seize indicateurs (dont seulement dix sont des indicateurs cliniques, pondérés à $60 \%$ du total) alors que, dans le cas britannique, la performance se définit par plus de cent-trente indicateurs (dont près de quatre-vingts indicateurs cliniques). Il y a alors deux risques : sous-définir la performance et la sur-définir. Dans les deux cas, la notion de qualité risque d'être diluée dans la notion de performance et plus encore dans les indicateurs eux-mêmes. L'une des conséquences pratiques de cette dissolution est l'effet de distorsion. Dans le cas du suivi des maladies chroniques par exemple, l'intéressement contribue à délaisser les tâches qui ne sont pas rétribuées [Bruin et al., 2011]. Il y a alors un risque que le médecin, en jouant l'objectif de performance, délaisse certaines activités non rétribuées et qui font la qualité de la relation médicale, comme par exemple le temps d'écoute du patient et globalement tout ce qui n'est pas mesurable tout en étant essentiel [Chanial, 2010]. L'écart entre ce que l'on entend par qualité des soins et les objectifs des mécanismes d'intéressement peut conduire à réduire la qualité tout en augmentant la performance !

Il est alors possible de mettre en évidence un décalage entre la notion de performance telle qu'elle est mesurée par les indicateurs (performance mesurée) et la notion de performance telle qu'elle est imaginée et souhaitée par le régulateur (performance idéale). Roski et al. [2003] ont testé l'effet du P4P sur la prise en charge du tabagisme. L'incitation était conditionnée à la distribution de documents sur les risques liés au tabac. Les auteurs ont observé une amélioration de la performance mesurée mais sans incitation réelle à adopter une attitude proactive du personnel de santé envers les patients (performance idéale). Cet effet est d'autant plus important que dans le domaine de la santé la performance 
idéale correspond en grande partie à la qualité des soins. Or cette notion est particulièrement difficile à définir, ce qui rend non moins difficile la construction d'indicateurs de performance (mesurée) performants (idéale).

\section{L'intérêt des médecins pour l'intéressement}

\section{De l'intérêt à la motivation}

Pour promouvoir le développement du P4P en France, certains auteurs ne s'appuient pas tant sur son efficacité prouvée que sur la (supposée) nécessité de revaloriser les revenus des médecins [Bras et Duhamel, 2008]. Or au vu de l'évaluation qui vient d'être faite, on peut se demander quelle est la pertinence de ce dispositif incitatif? La rationalité du dispositif n'est peut être pas là où on la pense. Que peuvent alors chercher les médecins dans la souscription où le rejet du P4P ? Afin de comprendre ces comportements face au P4P, nous pouvons recourir à l'analyse des motivations professionnelles.

La littérature grandissante en économie sur les motivations s'appuie notamment sur la distinction entre motivation intrinsèque (MI) et motivation extrinsèque (ME) issue de la psychologie sociale [Deci et Ryan, 2002]. Dans cette optique, une action est intrinsèquement motivée si elle est entreprise pour la satisfaction inhérente à son accomplissement. Elle est son propre but. Par exemple, lire un livre pour le plaisir de la lecture est une action relevant de la motivation intrinsèque. À l'inverse, la ME est une motivation instrumentale, au sens où la tâche est réalisée dans un but extérieur. Il s'agit d'obtenir une récompense ou d'éviter une sanction. Par exemple, lire un livre dans l'optique de la préparation d'un examen est une tâche extrinsèquement motivée. Dans ce cadre théorique, le $\mathrm{P} 4 \mathrm{P}$ est l'exemple typique de la ME car la tâche est rendue attrayante par l'intéressement financier. Seule la récompense est valorisée et non l'objectif lui-même. Si l'on prend l'exemple du CAPI, il faut prescrire des génériques non pas parce que c'est valorisant en soi mais parce que c'est profitable.

L'existence de plusieurs registres de motivation pose néanmoins un nouveau problème à l'économiste. En effet, l'hypothèse 
de substituabilité des motivations semble contraire au cadre économique standard où les motivations sont complémentaires [Da Silva, $2011 ; 2012$ a]. On dit qu'il y a crowding out effect (COE) lorsque l'introduction d'une ME, alors que l'action visée par l'incitation était auparavant intrinsèquement motivée, détruit les MI. Dans notre exemple, il y a COE si l'individu qui lisait un livre pour le plaisir perd son plaisir lorsqu'il est payé pour le lire. La ME a détruit la MI. Dans le cas de la santé, le P4P pourrait avoir pour effet de démotiver les médecins en évinçant leurs MI. L'incitation peut « désinciter ». Il s'agit alors, pour comprendre l'effet du P4P, de déterminer sous quelles conditions les motivations sont complémentaires (crowding in) ou substituables (crowding $o u t$ ). De cette littérature on peut retenir que, contrairement aux présupposés théoriques issus de la théorie économique standard, le P4P ne s'installe pas sur un terrain vierge de valeurs [Batifoulier, 2011]. Les médecins ont différents mobiles pour l'action, dont les MI font partie, et le succès ou le rejet du P4P est le fruit de la rencontre de ces motivations et du dispositif incitatif qui les conforte ou les modifie. Le rôle des caractéristiques sociodémographiques (âge, sexe, région d'exercice etc.), habituellement déterminant, ne permet pas d'expliquer l'adhésion ou le rejet du CAPI en France [Barley et al., 2011]. Les médecins sont diversement intéressés par l'intéressement et leur intérêt (ou désintérêt) pour l'intéressement dépend globalement de deux grandes caractéristiques [Da Silva, 2012b] :

- L'autonomie des pratiques : l'un des enseignements des études empiriques testant la motivation des médecins face à des mécanismes de $\mathrm{P} 4 \mathrm{P}$ est le rôle majeur de l'autonomie des pratiques. Il est possible de souligner le caractère contre-productif de ce type de mécanisme sur le comportement du médecin. On peut alors étayer l'hypothèse de crowding out effect. En réduisant l'autonomie du professionnel, l'intéressement aurait pour effet de réduire sa MI. Ce premier point n'est pas original tant il confirme les conclusions de la sociologie des professions. Le concept de profession à pratique prudentielle forgé par Champy [2009] met également en avant le rôle de l'autonomie. En plus de savoirs complexes dans un contexte d'incertitude, c'est l'impératif d'autonomie qui interdit au professionnel d'appliquer des règles normalisées. Dès lors, les mécanismes de P4P, par l'existence et le rôle central d'objectifs 
prédéfinis, semblent entrer en contradiction avec l'autonomie professionnelle.

- Le contexte institutionnel : soumis au même mode de rémunération (le $\mathrm{P} 4 \mathrm{P}$ ), deux contextes institutionnels différents peuvent avoir pour effet de produire des résultats contradictoires. Cette idée peut être illustrée par le contraste entre les cas français (CAPI) et britannique (QOF). La réticence plus virulente des médecins français au P4P peut ainsi s'expliquer par la justification et la construction institutionnelle de l'outil. Les objectifs initiaux et la relation entretenue entre médecins et tutelle sont en effet bien différents dans les deux cas [Bras, 2011]. Alors que le QOF avait pour objectif d'augmenter le budget du National Health Service, le CAPI s'inscrit dans le contexte d'une réduction des dépenses socialisées de santé. Ayant pour but de faire des économies, l'existence du champ « optimisation des prescriptions » cadre le dispositif comme un outil de réduction des dépenses. Or il apparaît contradictoire, pour de nombreux médecins, de réduire les dépenses et d'améliorer la qualité. Par ailleurs, il faut noter que les médecins britanniques entretiennent des rapports plus pacifiques avec la tutelle que les médecins français. L'une des explications tient au fait que le mécanisme de P4P britannique a été créé en partie par les représentants de médecins (ce qui n'est pas le cas en France).

La bonne réception des mécanismes de $\mathrm{P} 4 \mathrm{P}$ dépend donc de l'adéquation entre les valeurs qu'il véhicule et les valeurs médicales. La difficulté pour le régulateur vient alors du fait qu'il existe une pluralité de valeurs médicales parfois conflictuelles [Batifoulier, 2011]. L'exercice d'une médecine sociale peut ainsi s'opposer à une médecine libérale. S'il faut souligner que ni les unes ni les autres ne sont naturelles et qu'elles relèvent d'un construit social, le P4P n'est pas neutre. En effet, ce mécanisme d'intéressement véhicule, par construction, une rationalité marchande qui semble s'opposer à la conception traditionnelle de la relation patient-médecin. Le $\mathrm{P} 4 \mathrm{P}$ jette alors un flou sur la nature de l'activité médicale qui peut conduire à une situation de crowding out effect. On comprend alors mieux la dynamique de marchandisation des comportements [Batifoulier et Ventelou, 2003], et on voit, à travers l'étude des motivations, se brouiller l'analyse du comportement médical par le monisme de l'intérêt. 


\section{De quel intérêt parle-t-on?}

Les résultats de l'analyse des motivations médicales face au P4P font ressortir la diversité des mobiles de l'action chez les médecins. L'hypothèse de substituabilité des motivations fait même apparaître des difficultés à le conjuguer aux différents registres de motivations. Ces résultats semblent incompatibles avec les hypothèses habituelles du cadre économique standard [Da Silva, 2011]. Postuler qu'une action est intrinsèquement motivée implique qu'un individu puisse valoriser une activité en elle-même. Or en économie standard, le travail n'a pas de valeur, il est « désutile ». L'individu étant intéressé, seule la rémunération compte. L'intérêt intrinsèque pour la tâche semble une caractéristique superflue à l'analyse. L'hypothèse d'intéressement pose également un problème et mérite une discussion afin de comprendre quel est l'intérêt des médecins pour l'intéressement.

Dans l'optique d'une reconstruction théorique, nous pouvons nous appuyer sur les recherches d'Alain Caillé [2009]. Ses travaux sont fondés sur les découvertes anthropologiques fondamentales de Marcel Mauss dans l'Essai sur le don. Mauss y explique le don, et par là l'action humaine, par quatre dimensions premières et irréductibles « organisées en deux paires d'opposés : l'intérêt (égoïste) et le désintéressement (altruiste), l'obligation et la liberté » [Caillé, 20096 $]$. L'intérêt égoïste est à la fois intérêt matériel (lucratif) et intérêt de face (prestige), c'est le pôle utilitaire de l'action. Mais si l'action est intéressée, elle est également sujette à l'ouverture à l'autre, au désintéressement (ou encore à l'aimance, selon le néologisme d'Alain Caillé). À cette première opposition (intérêt - aimance), s'ajoute une seconde (liberté - obligation). En effet, l'action met également en œuvre la liberté individuelle (qui permet l'expression de la créativité) et l'obligation sociale (porteuse de sens). L'un des atouts de cette typologie, en plus d'échapper au monisme de l'intérêt, est d'insister sur l'irréductibilité de chacun des quatre pôles. Dans toute action, chaque pôle est actif ne serait-ce

6. Notons que, dans ses travaux, Alain Caillé préfère substituer aux intérêts « égoïstes » et « altruistes », qualificatifs aux contours incertains, ce qu'il appelle, respectivement, l'« intérêt pour soi » et l' ' intérêt pour autrui ». Cependant, par souci de simplicité, nous conserverons ici cette terminologie. 
que parce que toute obligation n'existe qu'en rapport à des libertés (et inversement) et que tout désintéressement (aimance) n'existe que par la sortie de l'intérêt (et inversement). Enfin, si au niveau positif ces quatre pôles irréductibles sont entremêlés pour expliquer l'action, au niveau normatif «il faut qu'il en soit ainsi » pour que le don permettent de nouer le lien social [Caillé, 2009]. Par exemple, le don purement altruiste insatisfait à la fois le donataire, qui est humilié, et le donateur, qui n'y trouve pas son compte. C'est pourquoi si le don est l'enchevêtrement des quatre pôles de l'action, il faut qu'il en soit ainsi pour faire société. La question du poids de chacun des pôles dans l'action et de leur articulation devient centrale.

Dans le cas du médecin, si l'on reprend les analyses des économistes standards, le seul motif d'action du médecin est l'intérêt égoïste (qu'il soit lucratif ou de face). On pense ici d'abord aux différentes théories visant à montrer que le médecin dispose d'un pouvoir discrétionnaire lui permettant d'adopter un comportement opportuniste au détriment du bien-être collectif. Mais les économistes standards ne méconnaissent pas la force de la contrainte et des comportements altruistes. Le problème est que ces motivations ne sont selon eux que des produits dérivés de l'intérêt égoïste. Il en est ainsi des modèles de contrainte de marché où le médecin adopte un comportement plutôt qu'un autre uniquement parce qu'il serait menacé par le pouvoir de marché des patients (celui de consulter un autre médecin et de faire jouer la concurrence). La contrainte, l'obligation, n'a pas de consistance propre et est rapportée à l'intérêt égoïste. Ce réductionnisme est le même que lorsqu'il s'agit d'intégrer les comportements désintéressés. Le désintéressement est modélisé dans le langage théorique standard de l'altruisme où le rapport à l'autre est représenté par l'interdépendance des fonctions d'utilité (médecin et patient). Dans ce cas, le médecin est altruiste parce que son bien-être est dépendant du bien-être du patient. Ce type d'altruisme est un altruisme intéressé et c'est toujours l'intérêt égoïste qui guide l'action.

Malgré l'incapacité de la théorie économique standard à modéliser les autres pôles de l'action, il est nécessaire de constater leur existence dans le comportement du médecin. Pour comprendre la pluralité des motivations et les intérêts pour le P4P, il faut alors recourir à des théories anti-utilitaristes de l'action médicale. Ainsi, 
dans le cadre de sa profession, le médecin est sujet à l'aimance (désintéressement). Il est un humaniste capable de faire don de soi au patient. Par ailleurs, son activité met en jeu sa liberté et sa créativité. Il peut éprouver un intérêt passionnel pour son métier. Ces deux nouveaux pôles de l'action ne sont pas réductibles à celui de l'intérêt égoïste. Le médecin peut apprécier le contact avec les patients et son métier sans que ces motivations soient le produit de l'intérêt égoïste. Enfin, et de façon tout à fait décisive, le médecin est également sujet, dans son activité, à l'obligation. Les codes de déontologie ou l'éthique sont là pour lui rappeler ses obligations vis-à-vis de la profession médicale, du patient et du reste du monde social [Hodgson, 2009]. Si elles obligent, ces obligations ne sont pas réductibles à la maximisation de l'utilité individuelle. Elles sont plutôt une manifestation du collectif porteuse de sens. Elles orientent l'action vers une finalité collectivement définie et à laquelle le médecin ne peut pas se soustraire.

Ces différents pôles de l'action, si l'on s'accorde pour ne pas tous les rabattre sur un seul qui serait déterminant « en dernière instance ", se prêtent bien à la comparaison avec la dichotomie des motivations étudiée précédemment. On peut associer les ME à l'intérêt égoïste. Dans ce cadre, l'activité du médecin est un instrument permettant d'accroître l'intérêt individuel. Tout se passe comme si le fait d'être médecin n'importait pas. Quel que soit le métier, c'est la satisfaction de l'intérêt égoïste qui compte. Les trois autres pôles de l'action, à l'inverse, correspondent aux MI. En effet, que l'on parle d'obligation, de liberté ou d'aimance, c'est l'activité qui est au centre des préoccupations. Le médecin est alors motivé par les impératifs déontologiques de la profession (obligation), la dimension créative ou passionnante de son activité (liberté) et la relation avec ses patients (aimance). Dans ce cadre, on peut réinterpréter l'intérêt des médecins pour l'intéressement. Pour comprendre l'effet caché du P4P, il faut adjoindre aux motivations utilitaires les motivations anti-utilitaires. Si l'on donne du crédit aux MI, on peut considérer que le médecin, en acceptant le $\mathrm{P} 4 \mathrm{P}$, cherche à améliorer la qualité de ses pratiques (cas britannique). Pour les mêmes raisons, il peut également refuser le $\mathrm{P} 4 \mathrm{P}$ (cas français). Par ailleurs, cette forme de motivation permet de prendre en compte la quête de reconnaissance. On peut enfin considérer que le médecin cherche à protéger ou à améliorer sa relation avec ses patients. 
Mais l'intervention publique, en promouvant des ME auparavant moins saillantes, les légitime au détriment des MI : c'est l'effet d'éviction (COE).

\section{Conclusion}

L'économie standard rapporte la régulation de l'activité médicale à la question des intérêts égoïstes. Naturellement opportuniste, le comportement du médecin ne peut être régulé que par le réagencement de ses intérêts instrumentaux. Pourtant cette hypothèse peine à expliquer les faibles résultats du $\mathrm{P} 4 \mathrm{P}$ ainsi que l'attitude des médecins face à ce type de mécanisme. On peut alors se demander si le médecin est exclusivement intéressé par l'intéressement? L'examen des motivations médicales a montré que tout n'est pas si simple. À côté du pôle utilitaire de l'action (l'intérêt égoïste), des motivations anti-utilitaires semblent particulièrement importantes pour les professionnels : définition de la bonne pratique, définition de la profession, problèmes éthiques et déontologiques, relation avec les patients, reconnaissance etc.

La problématique qui demeure en suspens, après ce travail de reconnaissance des intérêts anti-utilitaires, est celle de l'articulation des intérêts. Il s'agit certainement de la question primordiale. Les débats suscités par la mise en place du CAPI en France en témoignent. Comment doit-on envisager l'intégration des intérêts instrumentaux dans le cadre d'une profession où le désintéressement est un principe structurant? Dans quelle mesure la nécessaire conjugaison des intérêts se fait-elle au détriment (ou au profit) de l'un d'entre eux ? Est-ce que récompense rime avec reconnaissance?

\section{Références bibliographiques}

Barlet M., Bellamy V., Guillaumat-Tailliet F., Jakoubovitch S., 2011, «Médecins généralistes : que pensent-ils de leur rémunération? », Revue française des affaires sociales, $\mathrm{n}^{\circ} 2-3$, p. 123-155.

Batifoulier P., 2011, «Valeurs et conventions. Une économie politique de la santé », in Batifoulier P., Buttard A., Domin J.-P. (dir.), Santé et politiques 
sociales. Entre efficacité et justice, autour des travaux de Maryse Gadreau, Eska.

Batifoulier P., 2004, «L'économie contre l'éthique. Une tentative d'analyse économique de l'éthique médicale », Journal d'économie médicale, vol. 22, p. 163-176.

Batifoulier P., Ventelou B., 2003, « L'érosion de la part gratuite en médecine libérale. Discours économique et prophéties autoréalisatrices », Revue du MAUSS semestrielle, vol. 1, $\mathrm{n}^{\circ}$ 21, p. 313-329.

BRAS P.-L., 2011, « Le contrat d'amélioration des pratiques médicales (CAPI) à la lumière de l'expérience anglaise », Journal d'économie médicale, $\mathrm{n}^{\circ} 5$, vol. 29, p. 216-230.

Bras P.-L., Duhamel A., 2008, Rémunérer les médecins selon leurs performances : les enseignements des expériences étrangères, rapport de l'IGAS.

Bruni M., Nobilio L., UGolini C., 2008, « Economic incentives in general practice ; the impact of pay-for-participation and pay-for-compliance programs on diabetes care », Health Policy, ${ }^{\circ}$ 2-3, vol. 90, p. 140-148.

Bureau D., Mougeot M., 2007, Performance, incitations et gestion publique, Conseil d'analyse économique, La Documentation française, Paris.

CAIllé A., 2009, Théorie anti-utilitariste de l'action : fragments d'une sociologie générale, La Découverte, Paris.

Champy F., 2009, Sociologie des professions, PUF, Paris.

Chanial P., 2010, « Le new public management est-il bon pour la santé ? », Revue du MAUSS semestrielle, vol. 1, $\mathrm{n}^{\circ}$ 35, p. 135-50.

DA Silva N., 2012a, «Quelle éthique médicale ? Une relecture à partir de l'hypothèse de "crowding out effect" ", Éthique et Économique/Ethics and Economics, vol. 9, n 2, p. 65-84, < http://ethique-economique.net/>.

- 2012b, « Motivation et performance des médecins. Un examen de la littérature empirique », in Petrella F., Richez-Batesti N. (dir.), Travail, organisations et politiques publiques : quelle soutenabilité à l'heure de la mondialisation?, p. 371-587.

- 2011, «Motivations médicales et politiques d'incitations. La motivation intrinsèque contre la théorie de l'agence ? », Journal d'économie médicale, vol. $29, n^{\circ} 8$, p. 351-365.

De Bruin S., BaAn C., Struiss J., 2011, « Pay for performance in disease management : a systematic review of the literature », BMC, Health Service Research, vol. 11, $\mathrm{n}^{\circ} 272$.

DeCI E., RyAN R., 2002, Handbook of Self-Determination Research, University of Rochester Press, Rochester.

Doran T., Fullwood C., Gravelle H., Reeves D., Kontopantelis E., Hiroeh U., RolAND M., 2006, « Pay for performance programs in family practices in the United Kingdom », The New England Journal of Medicine, ${ }^{\circ} 355$, p. 375-384. 
Doran T., Fullwood C., Gravelle H., Roland M., 2008, « Exclusion of patients from pay for performance targets by English physicians », The New England Journal of Medicine, vol. 359, p. 274-284.

Galvin R., 2006, « Pay for performance : too much of a good thing ? A conversation with Martin Roland », Health Affairs, $n^{\circ}$ 5, vol. 25, p. 412-419.

Peckham S., Wallace A., 2010, «Pay for performance in primary care : what have we learnt ? », Quality in Primary Care, vol. 18, n² 2, p. 111-116.

Roski J., Jeddeloh R., An L., Lando H., Hannan P., Hall C., Zhu S. H., 2003, « The impact of financial incentives and a patient registry on preventive care quality : increasing provider adherence to evidence-based smoking cessation practice guidelines », Preventive Medicine, vol. 36, p. 291-299.

Van Herck P., De Smedt D., Annemans L., Remmen R., Rosenthal M., Sermeus W., 2010, « Systematic review : Effects, design choices, and context of pay-for-performance in health care », BMC, Health Services Research, vol. 10, $\mathrm{n}^{\circ} 1$. 